\title{
Arterial Thrombosis Related to Vascular Access Complication
}

National Cancer Institute

\section{Source}

National Cancer Institute. Arterial Thrombosis Related to Vascular Access Complication. NCl Thesaurus. Code C101338.

The formation of a thrombus in the artery as a direct result of an activity associated with vascular access. 